\title{
The Effect of Regulation Policy on Housing Prices in Areas Surrounding Beijing: An Empirical Study of Langfang Real Estate Regulation Policy
}

\author{
Zhenyang Qin
}

\author{
School of Government, Beijing Normal University, Beijing 100875, China
}

Keywords: Real estate market around Beijing; Real estate regulation policy; Policy effect; DID

\begin{abstract}
In the first half of 2016, in order to reduce the number of unsold homes, Chinese central government continued to implement stimulus policies in real estate market, making real estate market in first-tier and hot second-tier cities overheat. Meanwhile, the real estate markets in some satellite cities around core cities are also heating up rapidly, especially the area around Beijing. In April 2016, Langfang, a city close to Beijing, introduced real estate regulation policies to curb the excessive rise of housing prices. This paper applies difference in difference method (DID) to evaluate the actual effect of the real estate regulation policies in Langfang real estate market using the data from October 2015 to December 2016. The results show that house purchase restrictions in Langfang don't reduce the growth rate of new housing prices and expected growth rate of second-hand housing prices during sample period. The growth rate of second-hand house prices reduces by 2.827 percentage point during sample period. However, the absolute prices of second-hand house don't decrease.
\end{abstract}

\section{Introduction}

In the first half of 2016, China maintained loose credit and proactive fiscal policy. Compared with the recession in 2014 and the recovery in 2015, the property market grew rapidly, under the promotion of policies like destocking, dropping down payment and deed tax, and universal two-child policy. According to the data from National Bureau of Statistics, the sales area of commercial estate reached 643.02 million square meters from January to June in 2016, rising 27.9\% from a year earlier, while the sales volume reached 4.8682 trillion Yuan, increasing $42.1 \%$ from a year earlier. The prices of new houses in 70 large-and medium-sized cities were up by 1.56\%, 2.68\% and 3.83\% respectively month-on-month in the first three quarters. Meanwhile, the housing prices in satellite cities near some first-and second-tier cities raised greatly, some satellite cities around Beijing increasing most. In this paper, the property market in areas surrounding Beijing refers to markets in eight regions (counties), including Zhuozhou, Gu' an, Anci, Guangyang, Wuqing, Xianghe, Dachang and Sanhe. From June 2015 to Match 2016, the month-on-month rise of new housing prices fluctuated in these regions, but some places even surpassed Beijing in most months. In terms of the second-hand housing prices, the month-on-month increase exceeded Beijing in most months from June 2015 to Match 2016, the highest rise surpassing 10\%. Over the previous 10 months, the average increase of second-hand housing prices in areas surrounding Beijing was 2.22\%, much higher than that of Beijing (0.77\%), compared with a month earlier.

The main reasons of the rapid advance on housing prices in Beijing-surrounded areas are as follows. Recently, as Beijing's bearing capacity is gradually rich, many residential buildings are luxurious. However, the development costs of the cities near Beijing, such as Gu' an and Xianghe in Langfang and Wuqing in Tianjin, are relatively low, while most rigid and improved demand transfers to these regions. Then, the "Beijing-Tianjin-Hebei integration" strategy has balanced many aspects, including economy, the quality of social public service and urban infrastructure, in Beijing, Tianjin and Hebei. After finishing the rail transit, the cost of commuting would decline significantly. These measures will drive the rapid economic development in Beijing-surrounded areas and attract much investment. Thus, rigid and investment demand goes up, leading to the fast growth in housing prices in Beijing-surrounded regions. Under these circumstances, Langfang Government launched Suggestions on Improving the Housing Security System to Promote the Steady and Healthy Development of the Property Market in April 1st, 2016. Suggestions pointed out that, under the effect of policies like destocking, Langfang real estate has quickly risen since Match 2016, especially in counties (cities) around Beijing. It put forward a regulation 
that implements property-purchase limit and diversified housing credit. What's more, households with non-local residents are limited to one house and the proportion of the down payment is not less than $30 \%$.

Since 2010, China has conducted several rounds of property regulation to stabilize housing prices. However, there has been much debate over the effect of these regulation policies. Questions are also being raised frequently concerning why housing prices still remain high after regulation. Thus, does the purchase and credit limit work to restrain the growth of housing price? Chinese scholars evaluated the effect of real estate regulation policies in different cities of China. For example, Wang Xuefeng (2015) studied the temporal and spatial effects of purchase limit policy on the housing prices in Nanchang, which showed that there was no obvious difference of the housing price changes in limited and unlimited regions under the policy[1]; Li Yuxuan also believed that government's purchase restriction and credit policy restricted payment ratio is basically futile, based on empirical analysis of the dynamic panel data from 29 cities[2]. On the contrary, Han Yonghui et al. (2014) studied the monthly bill from 33 large-and medium-cities, which showed the purchase limit order curbed rising prices and significantly boosted the rational return[3]; Deng Baijun et al. (2014) drew similar conclusion[4].

There are four research methods. Wang Xuefeng (2015) and Qiao Kunyuan (2012) used Difference in Differences to estimate the effect of regulation policy[1, 5]; Han Yonghui et al. (2014), Deng Baijun et al. (2014), Zhang Derong and Zheng Xiaoting (2013) conduct Difference in Differences, after matching samples by propensity score, which improved the accuracy[3, 4, 6]; Zhang Jiantong et al. (2015) and Li Yuxuan (2015) used Regression Discontinuity Designs to research the effectiveness of the purchase limit order[7, 2]; Liu Xiaoyu et al. (2013), Jia Shenghua and Meng Zhenchao (2012) used VAR model to conduct empirical analysis on the market effect under purchase limit order[8,9]. Through reviewing literature, it can be seen that Difference in Differences is a common method to estimate policy effect. In research content, some questions are still up in the air. Firstly, scholars reached different conclusions on various samples. Therefore, the actual effect of property regulation policy may vary over time or place. Whether this regulation policy in Langfang will work or not still needs further study. Secondly, current researches mainly focus on the regulation policy in single city or large cities in China, instead of satellite cities. Out of the above considerations, this paper would analyze and demonstrate these two problems.

\section{Research Method and Data}

\subsection{Model and Variable}

\subsubsection{Setting treated and control groups}

The treated group includes individuals affected by policy, whereas the control one includes individuals without being affected. When the individual is treated, dummy variable $(d u)$ evaluates to 1 , otherwise 0 ; After the policy is launched, time ( $d t$ ) evaluates to 1 , otherwise 0 . In this research, the treated group includes Gu'an, Xianghe, Dachang and Sanhe in Langfang that implement purchase restriction policy, $d u=1$, while the control group includes Guangyang and Anci in Langfang, Zhuozhou and Wuqing in Tianjin without purchase limit policy, $d u=0$. The policy came into effect in May 2016, $d t=1$.

2.1.2 Calculating the effect of policy

According to the basic principle of Difference in Differences, if in natural experiment, we change treatment variable (policy) and remain other factors that influence dependent variable the same, average treatment effect

$\beta=(E(y \mid d u=1, d t=1)-E(y \mid d u=1, d t=0))-(E(y \mid d u=0, d t=1)-E(y \mid d u=0, d t=$ $0))$. Average treatment effect can also be estimated by econometric model. We can establish a regression model as Formula (1):

$$
Y=\beta_{0}+\beta_{1} d u+\beta_{2} d t+\beta_{3} d u * d t+\varepsilon
$$

Of these, $Y, d u, d t$ respectively represent rate of change in housing prices, dummy variable in policy and time, while $\varepsilon$ is random disturbance. Two dummy variables - $d u, d t$-divide the sample into four groups, namely the pre-treated group, post-treated group, pre-treated control group and post-treated 
control group. Therefore, average treatment effect: $(E(y \mid d u=1, d t=1)-E(y \mid d u=1, d t=0))-$ $(E(y \mid d u=0, d t=1)-E(y \mid d u=0, d t=0))=\beta_{2}+\beta_{3}-\beta_{2}=\beta_{3}$. Thus, in Formula (2), the estimated coefficient $\beta_{3}$ in $d t * d u$ measures the treatment influence of the policy. When policy is allocated by controlling variables, such as the population size in different regions and economic development, if we ignore the control variables in model, the estimators of Difference in Differences would be inaccurate. Instead, if taking these variables into consideration, we can eliminate the influence caused by them and receive pure policy effect. Therefore, we should add other control variables $(X)$ that affect $Y$ in model. The econometric model:

$$
Y=\beta_{0}+\beta_{1} d u+\beta_{2} d t+\beta_{3} d u * d t+\beta X+\varepsilon
$$

\subsubsection{Variables}

As the satellite cities of Beijing, surrounding areas shoulder much rigid demand from Beijing and foreign investment need. Thus, the fluctuation of housing prices in these regions may have much to do with the price and stock of housing in Beijing, as well as the transportation towards Beijing; then, these regions (counties) have their own endogenous demand, so their population size and economy may influence local housing prices. In view of these, we set an econometric model as Formula (3):

$$
Y=\beta_{0}+\beta_{1} d u+\beta_{2} d t+\beta_{3} d u * d t+\beta_{4} P o p+\beta_{5} P g d p+\beta_{6} \text { Distance }+\beta_{7} B e i P+\beta_{8} \text { BeiS }+\varepsilon
$$

$Y$ represents the month-on-month growth rate of new housing prices and second-hand housing prices in sample cities, $d u$ is the dummy variable of policy, $d t$ is dummy variable of time, Pop represents the population scale, $P g d p$ is the per capita GDP, Distance is the temporal distance between sample cities and Beijing, namely the commute time (average time of driving and public transportation), BeiP represents the month-on-month growth rate of housing price in Beijing, BeiS represents the stock of residential housing in Beijing and $\varepsilon$ is random error.

\subsection{Data}

From October 2015 to December 2016, the second-hand housing prices and expected prices in eight regions (counties) come from website (http://www.creprice.cn). CREIS database from China Index Academy provides the housing price and stock of Beijing. Gross population and per capita GDP in eight regions (counties) are from Statistical Yearbook of Baoding 2015, Yearbook of Economic Statistics Langfang 2015 and Statistical Yearbook of Tianjin 2015. And the commute time between these places and Beijing is calculated by Baidu Map.

\section{Empirical Analysis}

\subsection{Analysis on the effect of purchase restriction policy on new housing market}

According to Formula (3), this article conducts regression analysis with STATA14.0, taking growth rate of new housing as dependent variable. Result is shown in Tab 4. In column (1), before adding various control variables, the coefficient of variable did to measure policy effect is -1.230 . However, the regression coefficient is not statistically significant, which shows that the purchase restriction in Langfang has no impact on new housing prices. In column (2), after adding population size, per capita GDP and other control variables that may affect housing prices, the regression coefficient remains insignificant at level of $10 \%$, so does the regression coefficient of three control variables. It can be seen that the three control variables have nothing to do with the housing prices. In column (3), we add control variables related to Beijing, namely transportation, growth rate of housing price and stock of houses in Beijing. However, the coefficient of policy effect still remains insignificant at significance level of $10 \%$. Control variables related to Beijing have no influence on the growth rate of housing price in Beijing-surrounded regions. In summary, regulation policy in Langfang has no statistically significant effect on restraining the growth rate of new housing prices in regions implementing the policy; even we control internal and external factors. 
Table 1 Regression Results of Purchase Restriction Policy’s Effect on New housing Prices

\begin{tabular}{|l|c|c|c|}
\hline & \multicolumn{3}{|c|}{ Dependent Variable: $Y_{1}$} \\
\hline & $(1)$ & $(2)$ & $(3)$ \\
\hline & $1.440^{*}$ & $1.675^{*}$ & $2.077^{*}$ \\
\hline$d t$ & $(0.783)$ & $(0.962)$ & $(1.054)$ \\
\hline & $4.662^{* * *}$ & $4.662^{* * *}$ & $4.037 * * *$ \\
\hline did & $(1.041)$ & $(1.058)$ & $(1.440)$ \\
\hline & -1.230 & -1.230 & -1.230 \\
\hline Pop & $(1.538)$ & $(1.550)$ & $(1.558)$ \\
\hline & & 0.00682 & 0.0256 \\
\hline Pgdp & & $(0.0191)$ & $(0.0268)$ \\
\hline & & $-1.99 \mathrm{e}-05$ & $-5.98 \mathrm{e}-05$ \\
\hline Distance & $(2.54 \mathrm{e}-05)$ & $(5.08 \mathrm{e}-05)$ \\
\hline & & & -3.154 \\
\hline BeiP & & & $(3.129)$ \\
\hline & & & 0.0114 \\
\hline BeiS & & & $(0.0901)$ \\
\hline & & & -0.00400 \\
\hline Constant & & $(1.032)$ & $(0.00458)$ \\
\hline & & 120 & 14.63 \\
\hline Observations & 120 & 0.196 & $(11.00)$ \\
\hline R-squared & 0.191 & Note: robust standard errors are shown in brackets, & 0.207 \\
\hline
\end{tabular}

\subsection{Analysis on the effect of purchase restriction policy on second-hand housing market}

Second-hand housing prices consists of sales prices $Y_{2}$ and expected prices $Y_{3}$. The former could reflect the actual trading results in property market and the latter reflects buyers' expectation of second-hand housing prices. First of all, we do regression analysis using sales price as dependent variable. Result is shown in Tab 5. In column (1), before adding control variables, the coefficient of variable did to measure policy effect is -2.827 , which is significant at significance level of $10 \%$. It shows that the purchase limit policy reduces the growth rate of second-hand housing prices by 2.837 percentage point. In column (2), we add population size, per capita GDP and other control variables that may affect housing prices. The regression coefficient remains significant at level of $10 \%$. In column (3), we add control variables related to Beijing, namely transportation, growth rate of housing price and stock of houses in Beijing. Regression coefficient of policy effect remains significant at level of $10 \%$. In control variables, growth rate of housing price in Beijing has significant positive influence on growth rate of second-hand housing prices in Beijing-surrounded regions. For one percentage point of the growth rate of housing price in Beijing increased, the growth rate of second-hand house housing prices will go up by 0.308 percentage points in surrounding areas. Stock of residential housing in Beijing have no influence on the growth rate of second-hand housing prices in surrounding regions. After adding various control variables, $\mathrm{R}^{2}$ of the model increases greatly.

Then, we set the growth rate of second-hand housing expected price as dependent variable. In column (4), the coefficient of policy effect's variable did is -1.520 , but its regression coefficient remains insignificant at significance level of $10 \%$, namely the purchase restriction policy has no effect on buyers' expectation of the growth rate of second-hand house. In column (5), we add control variables like population size and per capita GDP, no changes occur in the regression coefficient of policy effect, and the test value of $t$ remains insignificant at level of $10 \%$. In column (6), regression coefficient of policy effect remains unchanged and the test value of $t$ is still insignificant at level of $10 \%$, after adding control variables related to Beijing. In control variables, growth rate of Beijing's housing price has significant positive influence on growth rate of second-hand house expected price in surrounding regions. For one 
percentage point of the growth rate of housing price in Beijing increased, the growth rate of second-hand housing prices will go up by 0.322 percentage point in surrounding areas.

Table 2 Regression Results of Purchase Restriction Policy's Effect on Second-hand housing Price

\begin{tabular}{|c|c|c|c|c|c|c|}
\hline & \multicolumn{3}{|c|}{ Dependent Variable: $Y_{2}$} & \multicolumn{3}{|c|}{ Dependent Variable: $Y_{3}$} \\
\hline & (1) & (2) & (3) & (4) & (5) & (6) \\
\hline \multirow[t]{2}{*}{$d u$} & $2.593 * * *$ & $1.816^{*}$ & 1.998* & 1.963 & 1.036 & 1.000 \\
\hline & $(0.906)$ & (1.050) & (1.048) & (1.937) & (1.943) & (1.792) \\
\hline \multirow[t]{2}{*}{$d t$} & $5.395^{* * *}$ & $5.395 * * *$ & $6.176^{* * *}$ & $4.493 * *$ & $4.493 * *$ & 3.425 \\
\hline & $(1.149)$ & $(1.155)$ & $(1.714)$ & $(1.885)$ & $(1.894)$ & $(2.774)$ \\
\hline \multirow[t]{2}{*}{ did } & $-2.827 *$ & $-2.827^{*}$ & $-2.827^{*}$ & -1.520 & -1.520 & -1.520 \\
\hline & (1.611) & (1.611) & (1.559) & (2.686) & (2.701) & (2.650) \\
\hline \multirow[t]{2}{*}{ Pop } & & -0.0285 & -0.0200 & & -0.0343 & -0.0359 \\
\hline & & $(0.0207)$ & $(0.0282)$ & & $(0.0344)$ & $(0.0492)$ \\
\hline \multirow[t]{2}{*}{$\operatorname{Pgdp}$} & & $1.93 \mathrm{e}-05$ & $1.40 \mathrm{e}-06$ & & $2.10 \mathrm{e}-05$ & $2.45 \mathrm{e}-05$ \\
\hline & & $(2.59 \mathrm{e}-05)$ & $(4.76 \mathrm{e}-05)$ & & $(4.32 \mathrm{e}-05)$ & $(8.15 e-05)$ \\
\hline \multirow[t]{2}{*}{ Distance } & & & -1.419 & & & 0.276 \\
\hline & & & $(3.076)$ & & & $(5.318)$ \\
\hline \multirow[t]{2}{*}{ BeiP } & & & $0.308 * * *$ & & & $0.322 *$ \\
\hline & & & $(0.0948)$ & & & $(0.192)$ \\
\hline \multirow[t]{2}{*}{ BeiS } & & & -0.00211 & & & -0.0135 \\
\hline & & & $(0.00552)$ & & & $(0.0115)$ \\
\hline \multirow[t]{2}{*}{ Constant } & $1.947 * * *$ & $2.873 * *$ & 8.183 & 2.041 & 3.263 & 14.57 \\
\hline & $(0.494)$ & $(1.195)$ & $(10.31)$ & $(1.337)$ & $(2.094)$ & $(20.73)$ \\
\hline Sample size & 120 & 120 & 120 & 120 & 120 & 120 \\
\hline $\mathrm{R}^{2}$ & 0.193 & 0.205 & 0.275 & 0.070 & 0.078 & 0.134 \\
\hline
\end{tabular}

All in all, purchase limit policy in Langfang has negative influence on the growth rate of second-hand housing prices in cities implementing the policy. It shows that the policy successfully restrains the surge of second-hand housing prices. However, the policy has no significant effect on the growth rate of second-hand housing expected prices, namely it fails to change buyers' expectation that second-hand house prices would rise. According to the regression coefficient and significance of control variables, the growth rate of Beijing's housing price does influence the second-hand housing prices in surrounding regions.

\section{Conclusion}

To study the actual effect of purchase restriction policy in Langfang, this paper determines the treated and control group based on Difference in Differences and choose internal and external control variables to construct econometric model. Then we conduct empirical analysis through data from 8 sample cities from October 2015 to December 2016. Results are as follows.

Firstly, the purchase restriction policy in Langfang has no significant effect on the growth rate of new housing prices. The reason may be that the implementation of purchase limit policy strengthens buyers' expectation that the house price would go up. Thus, instead of withdrawal, they enter the market, which leads to the rapid increase of new house price. In addition, developers may conduct methods like loans used for mortgage payments and lending identity card to avoid purchase restriction policy and reduce buyers' costs, which makes the policy fail to work.

Secondly, the purchase restriction policy decreases the growth rate of second-hand housing prices by 2.827 percentage point. It shows that the policy plays a role in restraining the surge of second-hand housing prices. However, the absolute price of second-hand house is still rising. Purchase limit policy has little effect on the growth rate of second-hand housing expected price, which means the absence of buyers' anticipation to the policy effect. The reason may be that the purchase limit policy in other regions fails to 
exert significant effect, which leaves a stereotype with buyers that the policy cannot restrain the increase of house price.

Thirdly, the house prices in Beijing-surrounded areas have much to do with Beijing's property market. The former shoulders much spilling demand in Beijing, so the increase of house prices is mainly caused by external factors instead of internal ones. The growth rate of housing prices in Beijing has positive influence on growth rate of new housing prices, second-hand housing price and second-hand housing expected price in Beijing-surrounded regions. Of these, the influence on the latter two is statistically significant. It indicates that the rise of Beijing property market will transfer to surrounding regions and make buyers anticipate that the surrounding areas will surge with it. The stock of Beijing's residential housing has negative influence on growth rate of new housing prices and second-hand housing prices in surrounding regions, all things being equal. It suggests that some buyers prefer to buy houses in Beijing when there is abundant storage. If supplies run tight, part of demand spills out to Beijing-surrounded regions, which bids up the house price.

\section{References}

[1] Wang Xuefeng. Land Spillover Effect and Temporal and Spatial Characteristics of the Effect of Housing Purchase Restriction Policy in Nanchang City [J]. China Land Science. 2015(06): 41-48.

[2] Li Yuxuan. Is the Exit of the Purchase and Credit Restriction Policy Reasonable?-an Empirical Analysis Based on the Dynamic Panel Data of 29 Cities [J]. Scientific Decision Making. 2015(07): 24-36.

[3] Han Yonghui, Huang Liangxiong, Zou Jianhua. On the effect of real estate "Limited Purchasing Order" policy [J]. Management of the Economy. 2014(04): 159-169.

[4] Deng Bojun, Li Zhongfei, Zhang Hao. Is the Limited Purchasing Order Effective in Regulating Housing Prices? [J]. Statistical Research. 2014(11): 50-57.

[5] Qiao Kunyuan. Does the Limited Purchasing Order Really Work? - Evidence from 70 cities in China [J]. Research on Economics and Management. 2012(12): 25-34.

[6] Zhang Derong, Zheng Xiaoting. Is the “Limited Purchasing Order” an Effective Policy to curb Housing Price? 1-An Empirical Study Based on 70 Large and Medium-Sized Cities [J]. The Journal of Quantitative \& Technical Economics. 2013(11): 56-72.

[7] Zhang Jiantong, Fang Chencheng, He Fang. Shanghai Real Estate Restricted Purchase and Loan Policy Evaluation: Research Based on Breakpoint Regression Design [J]. Scientific Decision Making. 2015(07): 1-23.

[8] Liu Xiaoyu, Xie Juanjuan, Zhao Heqin. Empirical Study on the Real Estate Market Performance under the Limited Purchasing Order [J]. Statistics \& Decision. 2013(04): 126-128.

[9] Jia Shenghua, Meng Zhenchao. The Effect and Its Sustainability of Real Estate Purchase Restriction Policy-An Empirical Study Based on the Relationship between Volume and Price Fluctuation in Beijing Commercial Residential Building Market [J]. China Economic Studies. 2012(05): 81-87. 